\title{
Algoritmo genético con tecnología Blockchain para reducir la entropía de una cadena de suministro
}

\author{
Juan José Miranda del Solar \\ jjmirand@ulima.edu.pe / Universidad de Lima. Lima, Perú \\ Max Guillermo Schwarz Díaz \\ mschwarz@ulima.edu.pe / Universidad de Lima. Lima, Perú
}

Recepción: 1-6-2018 / Aceptación: 20-8-2018

Resumen. La presente investigación desarrolla un algoritmo genético combinado con tecnología Blockchain para gestionar transacciones en una cadena de suministro reduciendo la entropía de la misma. La investigación utiliza un algoritmo genético para gestionar transacciones encriptadas usando el algoritmo sha256, y distribuirlas usando Blockchain para el flujo de gestión de las cadenas de suministros con componentes incrementales midiendo la entropía de Shannon de la cadena en Python 3.5 mediante técnicas de simulación. La investigación muestra cómo el uso de la programación genética combinada con tecnología Blockchain permite reducir la entropía de la cadena de suministro reduciendo con ello los costos y tiempos de transacción e incrementando los niveles de seguridad y confiabilidad en el proceso transaccional de toda la cadena.

PALABRAS CLAVE: algoritmos genéticos, Blockchain, cadena de suministro (SCM), entropía

\section{Blockchain-technology genetic algorithm for decreasing entropy in a supply chain}

Abstract. The present research develops a genetic algorithm combined with Blockchain technology to manage transactions in a supply chain reducing its entropy. The research uses a genetic algorithm to manage encrypted transactions using the sha256 algorithm and distribute them using Blockchain for the flow management of supply chains with incremental components by measuring Shannon's entropy of the chain in Python 3.5 using simulation techniques. The research shows how the use of genetic programming combined with Blockchain technology reduces the entropy of the supply chain, thereby reducing costs and transaction times and increasing the levels of security and reliability in the transaction process of the chain.

KEYWORDS: genetic algorithms, Blockchain, supply chain management (SCM), entropy 


\section{INTRODUCCIÓN}

El problema de la entropía en las cadenas de suministro (SCM) es un problema complejo que depende de diversos factores, como el número de intermediarios en la ruta desde el fabricante, mayorista, minorista, hasta llegar al cliente (Wever, Wognum, Tienekens y Omta, 2012; Tan y Cross, 2012; AbuKhousa, Al-Jaroodi, Lazarova-Molnar y Nader, 2014); la sincronización de los sistemas de información de los integrantes de la cadena (Horvath, 2001; Badenhorst, Maurer y Brevis-Landsberg, 2013; Al-Odeh, 2016), el capital intelectual acumulado en la cadena expresado como conocimiento, capacitación, entrenamiento o experiencia colaborativa de los integrantes de la cadena (Lin, Hung, Wu y Lin, 2002; Al-Mutawah, Lee y Cheung, 2009; Blome, Schoenherr y Eckstein, 2014; Williams, 2014), el marco regulatorio de las operaciones (Coppens, 2013; Huang, Yang y Wong, 2016), las restricciones tecnológicas, económicas e institucionales del contexto operativo (Kim, 2006; Yahia, 2009; AbuKhousa et al., 2014), el empuje de oferta del fabricante y la tracción de la demanda del mercado (Minculete y Olar, 2016; González-R, Framinan y Ruiz-Usano, 2013), la eficiencia en la gestión de los tiempos y costos transaccionales asociados a la velocidad del flujo y gestión de los activos (Ivanov, Sokolov y Kaeschel, 2011; Kim, Kim, Lim y Park, 2013), y la flexibilidad de adaptación de los componentes de la SCM (Fantazy, Kumar y Kumar, 2009; Candaci, Ngai y Moon, 2011) entre otros factores internos y externos del contexto de las operaciones de la misma. Estos factores combinados generan a su vez una amplia y compleja variabilidad y distorsión en la calidad, contenido, integridad y disponibilidad de la información que se procesa incrementando la entropía de la SCM como lo demuestran los trabajos de Isik (2009), Gerschberger, Engelhardt-Nowitzki, Kummer y Staberhofer (2012), Olatunde, Chan y Wang (2012).

El concepto de entropía de la información fue propuesto por Shannon (1948) en su Teoría matemática de la comunicación como una medida del desorden de los sistemas comunicacionales, a semejanza de la entropía fisicoquímica clásica, sobre la base del concepto de la unidad mínima de información transferible de un emisor a un receptor expresado como un múltiplo del logaritmo en base 2 (Isik, 2009). La entropía de Shannon refleja el desorden a partir de la probabilidad de transferencia del mensaje bajo el peso de su contenido, de manera que a medida que un sistema tiene más intermediarios, su entropía será mayor y por lo tanto será más desordenado y viceversa; la entropía se reduce con la menor intermediación posible (Olatunde, Chan y Wang, 2012). En ese contexto existen múltiples maneras de reducir la entropía de los flujos de información presentes en cualquier sistema, pero debido al avance de la potencia computacional sobre la base de la gestión de big data en la que operan las actividades empresariales, es necesario probar la factibilidad de uso de las nuevas tecnologías de información, en particular el uso de técnicas de inteligencia artificial y tecnologías Blockchain aplicadas a los procesos de negocio.

El ruido en las cadenas de suministro se presenta por la incertidumbre generada en el proceso de selección y transmisión de alternativas de transacción entre los miembros de la cadena, de manera tal que una cadena de suministro será más entrópica en tanto tenga más 
intermediarios y más cantidad de transacciones por resolver en la periferia de la toma de decisiones de cada nodo que la compone y, como indica Isik (2009), esto puede verse seriamente afectado si además la complejidad de las decisiones se vuelve variable y acepta múltiples selecciones como las previstas por Olatunde, Chan y Wang (2012), que pueden ser excluidas con reglas transaccionales predefinidas como en el caso de mercados perfectamente regulados como los previstos en el mercado internacional para importaciones y exportaciones de mercancías (ICONTEC, EUREPGAP y similares).

En el campo de la inteligencia artificial (IA) los algoritmos genéticos (AG) son algoritmos matemáticos de optimización inspirados en la genética humana, que tienen la capacidad de aprender y fijar genes en su mecanismo de operación para acercarse a soluciones de mayor rendimiento, descartando a la vez soluciones cuyos genes no conducen a mejorar los resultados. Normalmente los AG son inicializados con una solución básica, la cual es mejorada con modificaciones y revisada en resultados para admitirse o descartarse según su rendimiento frente a una función objetivo (Kahraman, Kaya y Cevikcan, 2011; Zhao, Zhang y Zhao, 2017). Los AG fueron propuestos por Holland (1975) y se han sofisticado con el avance de la potencia computacional, por lo que actualmente son utilizados en miles de aplicaciones con alta efectividad especialmente en ambientes que procesan grandes volúmenes de datos (big data). Los algoritmos genéticos permiten encontrar patrones de rendimiento entre grandes volúmenes de datos para descartar o acercarse a la búsqueda de mejores soluciones a partir del análisis recursivo, identificando características en los conjuntos de datos materia de prueba, de manera tal que se pueda establecer una clara regla que permita probar soluciones con mayor cercanía a la solución óptima esperable que se requiere alcanzar.

La tecnología Blockchain aparece hacia el 2009 y está fundamentada en la operación de una base de datos distribuida en la cual se acumulan bloques de transacciones que son confirmados por consenso en un ambiente de altos niveles de seguridad. Los bloques que se encadenan están constituidos por conjuntos de transacciones firmadas digitalmente para asegurar su integridad y que están disponibles para ser validadas por cualquiera de los nodos participantes en el sistema de la red distribuida. La aparición de esta nueva tecnología disruptiva plantea enormes retos al mercado porque representa una forma segura y confiable de transferir valor sin la necesidad de utilizar intermediarios, reduciendo los tiempos y costos de los procesos de manera radical (Nowinski y Kozma, 2017).

El uso de tecnología Blockchain en combinación con algoritmos genéticos es un tema relativamente nuevo en la literatura y recién ha sido abordado como posibilidad teórica en trabajos recientes por la necesidad de introducir técnicas de inteligencia artificial para el desarrollo de las nuevas aplicaciones de Blockchain, las cuales recién empiezan a ser desarrolladas pues la tecnología Blockchain ha sido principalmente aplicada a las criptomonedas y su aplicación a otras áreas de la industria aún está en un desarrollo incipiente, lo que genera espacio para numerosas investigaciones en el futuro (Woodside, Augustine Jr. y Giberson, 2017; Wu, Li, King, Zina, Wassick y Tazelaar, 2017). 
En el contexto de una cadena de suministro (SCM) ampliada independiente del número de intermediarios que existan o de la naturaleza del negocio en el cual se opera, como indican Badenhorst, Maurer y Brevis-Landsberg (2013), existe la enorme necesidad de lograr eficiencia y eficacia en el flujo transaccional que compone la cadena, para lo cual es necesario decidir el procesamiento entre miles de transacciones posibles que se presentan entre los nodos que interactúan, con la utilización de una técnica de inteligencia artificial que permita seleccionar las transacciones óptimas, lo cual será clave para reducir la entropía del sistema. Es ahí donde los algoritmos genéticos pueden hacer la diferencia en un primer momento, hasta lograr seleccionar el juego de transacciones de mayor rendimiento, menor costo y menor tiempo (mejor fitness) para el sistema.

A partir de ahí es necesario garantizar que las transacciones optimizadas seleccionadas no puedan ser alteradas circunstancialmente por los nodos, por lo que la tecnología Blockchain se constituye como una excelente alternativa para asegurar la eliminación de incertidumbre durante el flujo al interior de la SCM configurada.

\section{Metodología}

La investigación tiene como objetivo reducir la entropía de las SCM mediante el uso de un algoritmo genético combinado con tecnología Blockchain. Los algoritmos genéticos se utilizarán para priorizar las transacciones que operan en la SCM y la tecnología Blockchain para reducir la entropía del flujo transaccional que opera en la SCM. La entropía será medida como entropía de Shannon con Python 3.5 mediante técnicas de simulación en las SCM con distintos componentes incrementales. A las transacciones se les aplicará una función hash utilizando en este caso la función SHA256 (figura 1).

El algoritmo genético propuesto por los autores para la simulación de la SCM se describe como:

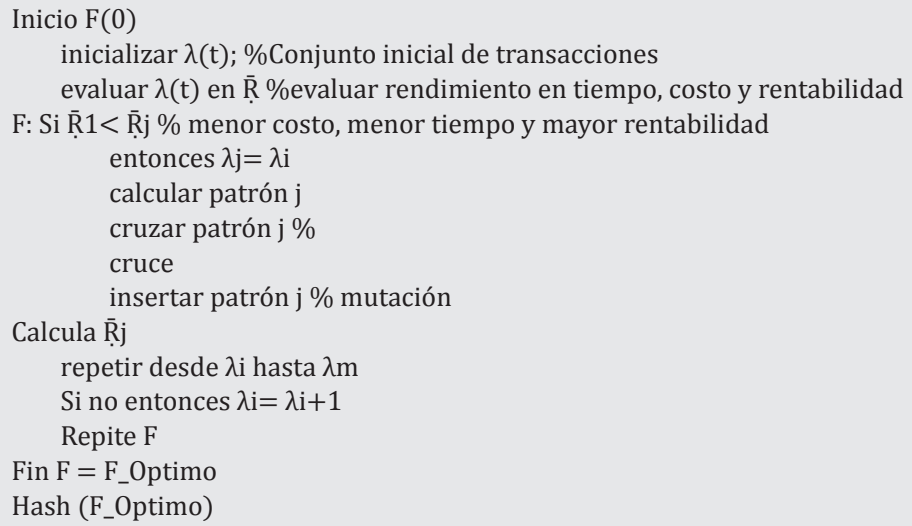


La entropía de Shannon se incorpora al algoritmo de la siguiente manera:

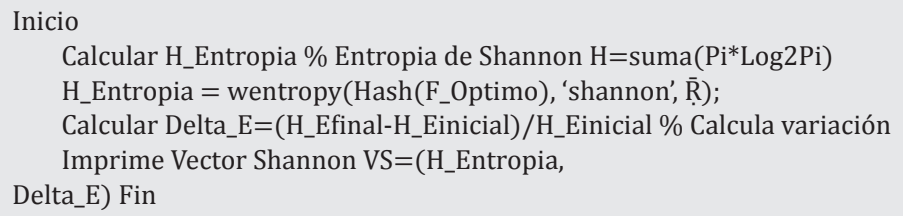

La configuración de Blockchain se incorpora al algoritmo de la siguiente manera:

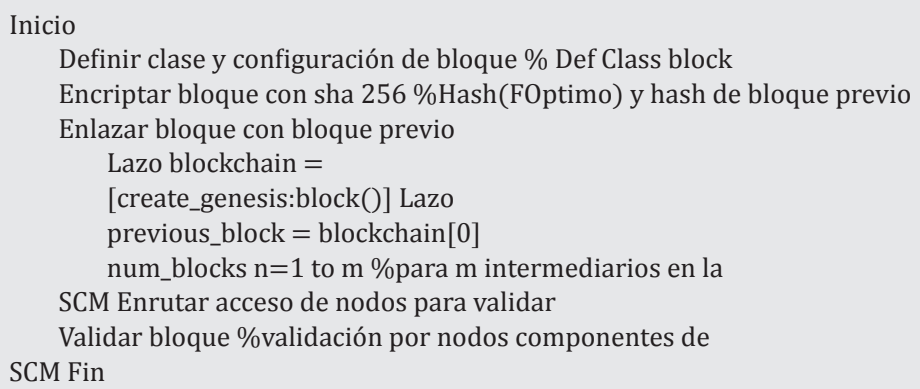

Para efectos de cálculo se utiliza Python 3.5 inicializando con una selección aleatoria de transacciones para su prueba con la cadena SCM en forma abierta para un número creciente de hasta " $m$ " intermediarios y se compara la entropía con el resultado de hacer el flujo con la incorporación del algoritmo propuesto para verificar las entropías de salida.

El procesamiento de datos incluye una selección aleatoria de " $n$ " transacciones posibles dentro de las que podrían definirse típicamente como transacciones probables a ser escogidas en la toma de decisiones empresariales al interior de la SCM, a partir de lo cual se procedió a la asignación aleatoria de fitness (combinación rentabilidad-costo-tiempo) por cada transacción, especificando el cromosoma madre como aquel que genera la mejor probabilidad de tener un fitness con mayor impacto para una generación de 10 millones de simulaciones sobre 1000 transacciones generadas aleatoriamente, con un ordenamiento resultante en función a la entropía de Shannon que genera cada transacción en su paso por el flujo de la SCM, utilizando un corte del $20 \%$ superior para la selección del juego de transacciones priorizadas que la organización debe elegir para reducir el ruido de su SCM. 


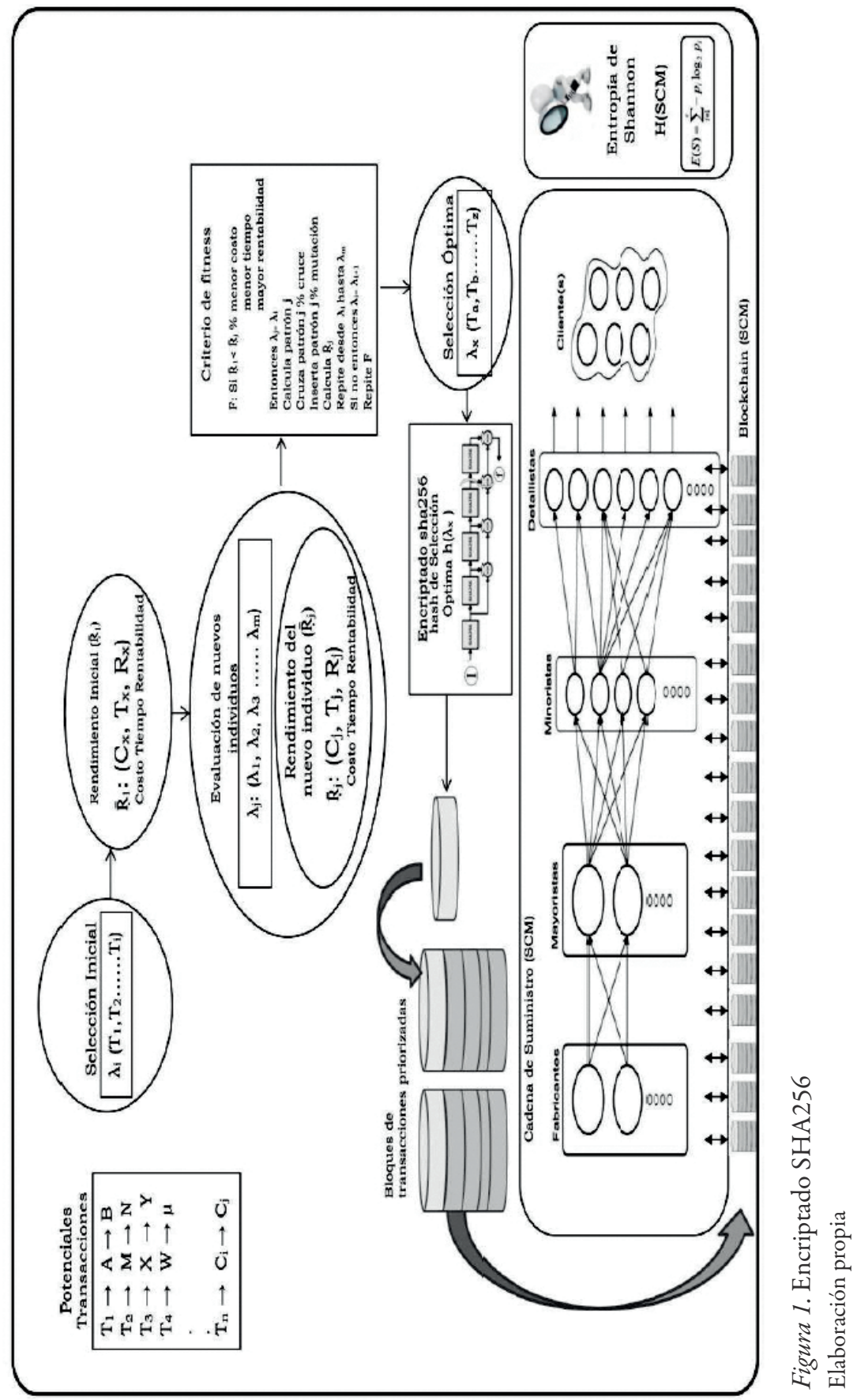




\section{Resultados}

Los resultados muestran los siguientes valores de fitness sobre la base de análisis de 10 millones de simulaciones, usando el algoritmo genético propuesto y una Blockchain privada de cuatro nodos simulada sobre Hyperledger Fabric versión 1.1:

Muestra SCM con diez intermediarios:

$[67,89,92,34,56,14,14,71,33,84]$

$[41,83,43,29,17,25,66,74,83,93]$

$[2,4,96,56,99,13,73,57,33,58]$

$[56,65,36,94,42,40,55,3,37,58]$

$[30,44,70,65,41,5,20,14,5,90]$

$[53,67,67,92,95,66,22,41,50,77]$

$[65,16,32,43,15,94,21,69,79,10]$

$[21,82,60,57,75,96,88,37,36,18]$

$[46,74,81,30,71,33,51,14,27,68]$

$[11,7,68,40,67,44,2,44,23,91]$

Muestra SCM con seis intermediarios:

$[15,34,63,12,60,24]$

$[90,79,22,95,2,5]$

$[13,23,14,43,13,6]$

$[33,3,8,12,67,4]$

$[62,77,2,52,8,45]$

$[31,87,55,93,13,11]$

$[60,100,93,82,12,35]$

$[12,30,36,3,81,65]$

$[38,81,51,21,6,37]$

$[79,81,65,83,20,42]$ 
Muestra SCM con tres intermediarios: $[48,56,94]$

$[6,78,14]$

$[54,28,7]$

$[44,92,14]$

$[16,67,78]$

$[81,19,82]$

$[74,7,14]$

$[12,14,98]$

$[19,22,18]$

$[15,2,7]$

Los valores optimizados de las transacciones para una SCM de 10 intermediarios son los siguientes:

0.04878512347946011

0.0490692630115289

0.050011113848945285

0.04879176665720224

0.049608380690151284

0.0495037040002993

0.048764900380000635

0.04884065760442752

0.04914354359659513

0.04935195562381178

Función fitness: DeltaEntropía $=0.0492$ 
Los valores optimizados de las transacciones para una SCM de seis intermediarios son los siguientes:

0.10460882471157217

0.1039429912716941

0.10356555109507334

0.10362554896434917

0.10498956495212682

0.10507075959013451

0.10463850504772856

0.10356460331729407

0.10459565852267928

0.10333992948491401

Función fitness: DeltaEntropía $=0.1041$

Los valores optimizados de las transacciones para una SCM de tres intermediarios son los siguientes:

0.2467253655388147

0.2477000603830717

0.24805288913740953

0.24645815477024427

0.24662242103729418

0.24742073689531716

0.24775772793018158

0.24707166462007746

0.2466148663142314

0.24725007378250927

Función fitness: DeltaEntropía $=0.2471$ 


\section{Conclusiones}

La investigación demuestra que la entropía de Shannon para el caso del algoritmo genético sobre la base de 10 intermediarios en la cadena, considerando 1000 muestras en 1000 iteraciones y el corte al $20 \%$ de los mejores, es de $4,92 \%$ de reducción de la entropía. De igual forma se demuestra que luego de reducir el número de intermediarios al $60 \%$ se logra una reducción de la entropía de hasta 10,41 \% y si la reducción de intermediarios se produce hasta un $30 \%$ se logra una reducción de la entropía de hasta un 24,71\%. Esto significa que consistentemente, a medida que se reducen los intermediarios en la SCM, las reducciones en la entropía son incrementalmente mayores, lo cual es consistente con la realidad empresarial; más aún si se tiene en cuenta que la cadena mínima conformable de una SCM real incluye un mínimo de tres intermediarios; entonces podemos concluir que la máxima reducción de entropía lograble converge a 24,71\% por el efecto directo de la aplicación del algoritmo genético en la selección de las transacciones y de un 99,99\% en el flujo al utilizar la tecnología Blockchain puesto que esta, por su configuración de diseño, anula el ruido de manera estructural, generando una optimización del fitness con el que se mide la SCM global.

La investigación demuestra consistentemente que el uso del algoritmo genético propuesto permite una reducción de la entropía de Shannon de la SCM de hasta un 24,71 \% en la etapa de priorización transaccional y de un 99,99 \% en la etapa de distribución de la información en los SCM usando tecnología Blockchain. De igual forma, a partir de los resultados puede apreciarse que la función de rendimiento mantiene una dependencia sensitiva al número de intermediarios que componen la cadena en mayor proporción que los otros factores estudiados.

La utilización red Blockchain, donde cada intermediario de la cadena es un peer de dicha red, debidamente identificado con su llave pública usando algoritmos de firma digital de curva elíptica (ECDSA), donde cada intermediario almacena su llave privada para asegurar la autenticidad y veracidad de la información a transferir como parte del flujo y finalmente aplicando funciones hash a este contenido junto con el hash del bloque anterior, nos permite un registro distribuido, seguro, inmutable y a prueba de manipulaciones de la información de las transacciones para cada uno de los intermediarios que conforman la SCM, logrando un 99,99\% de confiabilidad y reducción del ruido en el flujo de la información.

Las principales limitaciones de la investigación se presentan en la selección del algoritmo genético a utilizarse en la simulación (dada la amplia variedad de opciones disponibles) y en la naturaleza compleja de la composición de la SCM que requiere evaluarse, ya que no se trata solamente del tamaño de la cadena sino de la complejidad, aprendizaje, cultura y experiencia de cada componente o intermediario que conforma la SCM. Estas consideraciones escapan al alcance de la presente investigación y requieren ser planteadas para investigar con mayor detalle el potencial rendimiento de la SCM en futuras investigaciones. 


\section{REFERENCIAS}

AbuKhousa, E., Al-Jaroodi, J., Lazarova-Molnar, S., y Nader, M. (2014). Simulation and modeling efforts to support decision making in healthcare supply chain management. The Scientific World Journal. DOI: 10.1155/2014/354246

Al-Mutawah, K., Lee, V., y Cheung, Y. (2009). A new multi-agent system framework for tacit knowledge management in manufacturing supply chains. Journal of Intelligent Manufacturing 20(5), 593-610. DOI:10.1007/s10845-008-0142-0

Al-Odeh, M. (2016). Supply chain information systems technologies and management strategies in northern minnesota. Journal of Supply Chain Management Systems 5(2).

Badenhorst, J. A., Maurer, C., y Brevis-Landsberg, T. (2013). Developing measures for the evaluation of information flow efficiency in supply chains. Journal of Transport and Supply Chain Management 7(1). DOI:10.4102/jtscm.v7i1.88

Blome, C., Schoenherr, T., y Eckstein, D. (2014). The impact of knowledge transfer and complexity on supply chain flexibility: A knowledge-based view. International Journal of Production Economics 147, pp. 307-316. DOI:10.1016/j.ijpe.2013.02.028

Candaci, Y. Y., Ngai, E. W. T. y Moon, K.-L. (2011). Supply chain flexibility in an uncertain environment: exploratory findings from five case studies. Supply Chain Management: An International Journal 16(4), pp. 271-283.

Coppens, D. (2013). How special is the special and differential treatment under the SCM agreement? A legal and normative analysis of WTO subsidy disciplines on developing countries. World Trade Review 12(1), 79-109.DOI:10.1017/S1474745612000493

Fantazy, K. A., Kumar, V. y Kumar, U. (2009). An empirical study of the relationships among strategy, flexibility and performance in the supply chain context. Supply Chain Management: An International Journal 14(3), pp. 177-188.

Gerschberger, M., Engelhardt-Nowitzki, C., Kummer, S., y Staberhofer, F. (2012). A model to determine complexity in supply networks. Journal of Manufacturing Technology Management 23(8), 1015-1037. DOI:10.1108/17410381211276853

González-R. P. L., Framinan, J. M., y Ruiz-Usano, R. (2013). A methodology for the design and operation of pull-based supply chains. Journal of Manufacturing Technology Management 24(3), 307-330. DOI:10.1108/17410381311318855

Holland, J. H. (1975). Adaptation in natural and artificial systems: an introductory analysis with applications to biology, control, and artificial intelligence. Boston: MIT Press.

Horvath, L. (2001). Collaboration: The key to value creation in supply chain management. Supply Chain Management 6(5), 205-207. 
Huang, Y., Yang, M., y Wong, Y. (2016). Institutional pressures, resources commitment, and returns management. Supply Chain Management 21(3), 398-416.

Isik (2009). An entropy based approach for measuring complexity in supply chains. International Journal of Production Research, 48(12), 3681-3696.

Ivanov, D., Sokolov, B., y Kaeschel, J. (2011). Integrated supply chain planning based on a combined application of operations research and optimal control. Central European Journal of Operations Research 19(3), 299-317. DOI:10.1007/s10100-010-0185-0

Kahraman, C., Kaya, I., y Cevikcan, E. (2011). Intelligence decision systems in enterprise information management. Journal of Enterprise Information Management 24(4), pp. 360-379. DOI:10.1108/17410391111148594

Kim, K., Kim, T., Lim, D., y Park, H. M. (2013). Managing the supply and demand uncertainty in workforce recruitment: Planned or just-in-time acquisition. The Journal of the Operational Research Society 64(11), 1654-1663. DOI:10.1057/jors.2012.152

Kim, S. W. (2006). Effects of supply chain management practices, integration and competition capability on performance. Supply Chain Management 11(3), pp. 241248. DOI:10.1108/13598540610662149

Lin, C., Hung, H. C., Wu, J. Y., y Lin, B. (2002). A knowledge management architecture in collaborative supply chain. Journal of Computer Information Systems 42(5), pp. 83-94. DOI:10.1080/08874417.2002.11647612

Minculete, G., y Olar, P. (2016). "Push" and "Pull" systems in supply chain management. correlative approaches in the military field. Journal of Defense Resources Management $7(2), 165-172$.

Nowiński, W., y Kozma, M. (2017). How can blockchain technology disrupt the existing business models? Entrepreneurial Business and Economics Review 5(3), 173-188. DOI:10.15678/EBER.2017.050309

Olatunde, A. D., Chan, H. K., y Wang, X. (2012). Entropy assessment of supply chain disruption. Journal of Manufacturing Technology Management 23(8), pp. 998-1014. DOI: $10.1108 / 17410381211276844$

Schwarz-Díaz, M. G. (2014). Una medida de la incertidumbre basada en la entropía para cuantificar el impacto de la pérdida de información de los sistemas de gestión. Paideia XXI 4(5), pp. 46-56.

Shannon, C. E. (1948). A mathematical theory of communication. The Bell System Technical Journal, 27(3). 
Tan, K., y Cross, J. (2012). Influence of resource-based capability and inter-organizational coordination on SCM. Industrial Management \& Data Systems 112(6), pp. 929-945. DOI:10.1108/02635571211238527

Yahia, Z. M. (2009). The collaborative supply chain. Assembly Automation 29(2), pp. 127-136. DOI:10.1108/01445150910945589

Wever, M., Wognum, P. M., Trienekens, J. H., y Omta, S. W. F. (2012). Supply chain-wide consequences of transaction risks and their contractual solutions: towards an extended transaction cost economics framework. Journal of Supply Chain Management 48(1), pp. 73-91.

Williams, A.J. (2014). Transporting tacit supply knowledge in competitive environments: a storytelling perspective. International Journal of Procurement Management 7(5), pp. 622-638. DOI:10.1504/IJPM.2014.064622

Woodside, J. M., Augustine Jr., F. K., y Giberson, W. (2017). Blockchain technology adoption status and strategies. Journal of International Technology and Information Management 26(2), pp. 65-93.

Wu, H., Li, Z., King, B., Zina, B. M., Wassick, J., y Tazelaar, J. (2017). A distributed ledger for supply chain physical distribution visibility. Information 8(4), pp. 137. DOI:10.3390/ info8040137

Zhao, Q., Zhang, C., y Zhao, Z. (2017). SCM: A method to improve network service layout efficiency with network evolution. PLoS One 12(12). DOI:10.1371journal. pone.0189336 
